\title{
The Complexity of Codiagnosability for Discrete Event and Timed Systems
}

\author{
Franck Cassez ${ }^{\star}$ \\ National ICT Australia \& CNRS \\ The University of New South Wales \\ Sydney, Australia
}

\begin{abstract}
In this paper we study the fault codiagnosis problem for discrete event systems given by finite automata (FA) and timed systems given by timed automata (TA). We provide a uniform characterization of codiagnosability for FA and TA which extends the necessary and sufficient condition that characterizes diagnosability. We also settle the complexity of the codiagnosability problems both for FA and TA and show that codiagnosability is PSPACE-complete in both cases. For FA this improves on the previously known bound (EXPTIME) and for TA it is a new result. Finally we address the codiagnosis problem for TA under bounded resources and show it is 2EXPTIME-complete.
\end{abstract}

\section{Introduction}

Discrete-event systems [16]17] (DES) can be modelled by finite automata (FA) over an alphabet of observable events $\Sigma$.

The fault diagnosis problem is a typical example of a problem under partial observation. The aim of fault diagnosis is to detect faulty sequences of the DES. The assumptions are that the behavior of the DES is known and a model of it is available as a finite automaton over an alphabet $\Sigma \cup\{\tau, f\}$, where $\Sigma$ is the set of observable events, $\tau$ represents the unobservable events, and $f$ is a special unobservable event that corresponds to the faults: this is the original framework introduced by M. Sampath et al. [18, and the reader is referred to this paper for a clear and exhaustive introduction to the subject. A faulty sequence is a sequence of the DES containing an occurrence of event $f$. An observer which has to detect faults, knows the specification/model of the DES, and it is able to observe sequences of observable events. Based on this knowledge, it has to announce whether an observation it makes (in $\Sigma^{*}$ ) was produced by a faulty sequence (in $\left.(\Sigma \cup\{\tau, f\})^{*}\right)$ of the DES or not. A diagnoser (for a DES) is an observer which observes the sequences of observable events and is able to detect whether a fault event has occurred, although it is not observable. If a diagnoser can detect a fault at most $\Delta$ step 11 after it has occurred, the DES is said to be

\footnotetext{
* Author supported by a Marie Curie International Outgoing Fellowship within the 7th European Community Framework Programme.

${ }^{1}$ Steps are measured by the number of transitions in the DES.
} 
$\Delta$-diagnosable. It is diagnosable if it is $\Delta$-diagnosable for some $\Delta \in \mathbb{N}$. Checking whether a DES is $\Delta$-diagnosable for a given $\Delta$ is called the bounded diagnosability problem; checking whether a DES is diagnosable is the diagnosability problem.

Checking diagnosability for a given DES and a fixed set of observable events can be done in polynomial time using the algorithms of 13/21. If a diagnoser exists there is a finite state one. Nevertheless the size of the diagnoser can be exponential as it involves a determinization step. The extension of this DES framework to timed automata (TA) has been proposed by S. Tripakis in [19], and he proved that the problem of checking diagnosability of a timed automaton is PSPACE-complete. In the timed case, the diagnoser may be a Turing machine. The problem of checking whether a timed automaton is diagnosable by a diagnoser which is a deterministic timed automaton was studied by P. Bouyer et al. [5].

Codiagnosability generalizes diagnosability by considering decentralized architectures. Such decentralized architectures have been introduced in [10] and later refined in 20[15]. In these architectures, local diagnosers (with their own partial view of the system) can send to a coordinator some information, summarizing their observations. The coordinator then computes a result from the partial results of the local diagnosers. The goal is to obtain a coordinator that can detect the faults in the system. When local diagnosers do not communicate with each other nor with a coordinator (protocol 3 in [10]), the decentralized diagnosis problem is called codiagnosis [15[20]. In this case, codiagnosis means that each fault can be detected by at least one local diagnoser. In the paper [15], codiagnosability is considered and an algorithm to check codiagnosability is presented for discrete event systems (FA). An upper bound for the complexity of the algorithm is EXPTIME. In 20, the authors consider a hierarchical framework for decentralized diagnosis. In [3] a notion of robust codiagnosability is introduced, which can be thought of as a fault tolerant (local diagnosers can fail) version of codiagnosability.

None of the previous papers has addressed the codiagnosability problems for timed automata. Moreover, the exact complexity of the codiagnosis problems is left unsettled for discrete event systems (FA).

Our Contribution. In this paper, we study the codiagnosability problems for FA and TA. We settle the complexity of the problems for FA (PSPACE-complete), improving on the best known lower bound (EXPTIME). We also address the codiagnosability problems for TA and provide new results: algorithms to check codiagnosability and also codiagnosability under bounded resources. Our contribution is both of theoretical and practical interests. The algorithms we provide are optimal, and can also be implemented using standard model-checking tools like SPIN 12 for FA, or UPPAAL [4] for TA. This means that very expressive languages can be used to specify the systems to codiagnose and very efficient implementations and data structures are readily available.

Organisation of the Paper. Section 2 recalls the definitions of finite automata and timed automata. We also give some results on the Intersection Emptiness 
Problems (section 2.6) that will be used in the next sections. Section 3 introduces the fault codiagnosis problems we are interested in, and a necessary and sufficient condition that characterizes codiagnosability for FA and TA. Section 4 contains the first main results: optimal algorithms for the codiagnosability problems for FA and TA. Section [5] describes how to synthesize the codiagnosers and the limitations of this technique for TA. Section 6 is devoted to the codiagnosability problem under bounded resources for TA and contains the second main result of the paper.

\section{Preliminaries}

$\Sigma$ denotes a finite alphabet and $\Sigma_{\tau}=\Sigma \cup\{\tau\}$ where $\tau \notin \Sigma$ is the unobservable action. $\mathbb{B}=\{$ TRUE, FALSE $\}$ is the set of boolean values, $\mathbb{N}$ the set of natural numbers, $\mathbb{Z}$ the set of integers and $\mathbb{Q}$ the set of rational numbers. $\mathbb{R}$ is the set of real numbers and $\mathbb{R}_{\geq 0}$ (resp. $\mathbb{R}_{>0}$ ) is the set of non-negative (resp. positive) real numbers. We denote tuples (or vectors) by $\bar{d}=\left(d_{1}, \cdots, d_{k}\right)$ and write $\bar{d}[i]$ for $d_{i}$.

\subsection{Clock Constraints}

Let $X$ be a finite set of variables called clocks. A clock valuation is a mapping $v: X \rightarrow \mathbb{R}_{\geq 0}$. We let $\mathbb{R}_{\geq 0}^{X}$ be the set of clock valuations over $X$. We let $\overline{0}_{X}$ be the zero valuation where all the clocks in $X$ are set to 0 (we use $\overline{0}$ when $X$ is clear from the context). Given $\delta \in \mathbb{R}, v+\delta$ is the valuation defined by $(v+\delta)(x)=v(x)+\delta$. We let $\mathcal{C}(X)$ be the set of convex constraints on $X$, i.e., the set of conjunctions of constraints of the form $x \bowtie c$ with $c \in \mathbb{Z}$ and $\bowtie \in\{\leq,<,=,>, \geq\}$. Given a constraint $g \in \mathcal{C}(X)$ and a valuation $v$, we write $v \models g$ if $g$ is satisfied by the valuation $v$. We also write $\llbracket g \rrbracket$ for the set $\{v \mid v \models g\}$. Given a set $R \subseteq X$ and a valuation $v$ of the clocks in $X, v[R]$ is the valuation defined by $v[R](x)=v(x)$ if $x \notin R$ and $v[R](x)=0$ otherwise.

\subsection{Timed Words}

The set of finite (resp. infinite) words over $\Sigma$ is $\Sigma^{*}$ (resp. $\Sigma^{\omega}$ ) and we let $\Sigma^{\infty}=\Sigma^{*} \cup \Sigma^{\omega}$. A language $L$ is any subset of $\Sigma^{\infty}$. A finite (resp. infinite) timed word over $\Sigma$ is a word in $\left(\mathbb{R}_{\geq 0} \cdot \Sigma\right)^{*} \cdot \mathbb{R}_{\geq 0}\left(\operatorname{resp} .\left(\mathbb{R}_{\geq 0} \cdot \Sigma\right)^{\omega}\right)$. Duration $(w)$ is the duration of a timed word $w$ which is defined to be the sum of the durations (in $\mathbb{R}_{\geq 0}$ ) which appear in $w$; if this sum is infinite, the duration is $\infty$. Note that the duration of an infinite word can be finite, and such words which contain an infinite number of letters, are called Zeno words. We let Unt(w) be the untimed version of $w$ obtained by erasing all the durations in $w$. An example of untiming is $\operatorname{Unt}(0.4 a 1.0 \quad b 2.7 c)=a b c$. In this paper we write timed words as $0.4 a 1.0 \quad b 2.7 c \cdots$ where the real values are the durations elapsed between two letters: thus $c$ occurs at global time 4.1 . 
$T W^{*}(\Sigma)$ is the set of finite timed words over $\Sigma, T W^{\omega}(\Sigma)$, the set of infinite timed words and $T W(\Sigma)=T W^{*}(\Sigma) \cup T W^{\omega}(\Sigma)$. A timed language is any subset of $T W(\Sigma)$.

Let $\boldsymbol{\pi}_{\Sigma^{\prime}}$ be the projection of timed words of $T W(\Sigma)$ over timed words of $T W\left(\Sigma^{\prime}\right)$. When projecting a timed word $w$ on a sub-alphabet $\Sigma^{\prime} \subseteq \Sigma$, the durations elapsed between two events are set accordingly: for instance for the timed word $0.4 a 1.0 \quad b 2.7 c$, we have $\pi_{\{a, c\}}\left(\begin{array}{llllll}0.4 & a & 1.0 & b & 2.7 & c\end{array}\right)=0.4 a 3.7 c$ (note that projection erases some letters but keep the time elapsed between two letters). Given a timed language $L$, we let $\operatorname{Unt}(L)=\{\operatorname{Unt}(w) \mid w \in L\}$. Given $\Sigma^{\prime} \subseteq \Sigma, \boldsymbol{\pi}_{\Sigma^{\prime}}(L)=\left\{\boldsymbol{\pi}_{\Sigma^{\prime}}(w) \mid w \in L\right\}$.

\subsection{Timed Automata}

Timed automata are finite automata extended with real-valued clocks to specify timing constraints between occurrences of events. For a detailed presentation of the fundamental results for timed automata, the reader is referred to the seminal paper of R. Alur and D. Dill [2].

Definition 1 (Timed Automaton). A Timed Automaton $A$ is a tuple $\left(L, l_{0}\right.$, $\left.X, \Sigma_{\tau}, E, \operatorname{Inv}, F, R\right)$ where:

- L is a finite set of locations;

- $l_{0}$ is the initial location;

- $X$ is a finite set of clocks;

$-\Sigma$ is a finite set of actions;

- $E \subseteq L \times \mathcal{C}(X) \times \Sigma_{\tau} \times 2^{X} \times L$ is a finite set of transitions; in a transition $\left(\ell, g, a, r, \ell^{\prime}\right), g$ is the guard, a the action, and $r$ the reset set; as usual we often write a transition $\ell \stackrel{g, a, r}{\longrightarrow} \ell^{\prime}$;

- Inv $\in \mathcal{C}(X)^{L}$ associates with each location an invariant; as usual we require the invariants to be conjunctions of constraints of the form $x \preceq c$ with $\preceq \in$ $\{<, \leq\}$;

$-F \subseteq L$ (resp. $R \subseteq L$ ) is the final (resp. repeated) set of locations.

The size of a TA $A$ is denoted $|A|$ and is the size of the clock constraints i.e., the size of the transition relation $E$. A state of $A$ is a pair $(\ell, v) \in L \times \mathbb{R}_{>0}^{X}$. A run $\varrho$ of $A$ from $\left(\ell_{0}, v_{0}\right)$ is a (finite or infinite) sequence of alternating delay and discrete moves:

$$
\varrho=\left(\ell_{0}, v_{0}\right) \stackrel{\delta_{0}}{\longrightarrow}\left(\ell_{0}, v_{0}+\delta_{0}\right) \stackrel{a_{0}}{\longrightarrow}\left(\ell_{1}, v_{1}\right) \cdots \stackrel{a_{n-1}}{\longrightarrow}\left(\ell_{n}, v_{n}\right) \stackrel{\delta_{n}}{\longrightarrow}\left(\ell_{n}, v_{n}+\delta_{n}\right) \cdots
$$

s.t. for every $i \geq 0$ :

$-v_{i}+\delta \models \operatorname{Inv}\left(\ell_{i}\right)$ for $0 \leq \delta \leq \delta_{i}$;

- there is some transition $\left(\ell_{i}, g_{i}, a_{i}, r_{i}, \ell_{i+1}\right) \in E$ s.t. $:(i) v_{i}+\delta_{i} \models g_{i},(i i)$ $v_{i+1}=\left(v_{i}+\delta_{i}\right)\left[r_{i}\right]$. 
The set of finite (resp. infinite) runs in $A$ from a state $s$ is denoted $\operatorname{Runs}^{*}(s, A)$ (resp. Runs $\left.{ }^{\omega}(s, A)\right)$. We let Runs ${ }^{*}(A)=\operatorname{Runs}^{*}\left(s_{0}, A\right)$, Runs $^{\omega}(A)=\operatorname{Runs}^{\omega}\left(s_{0}, A\right)$ with $s_{0}=\left(l_{0}, \overline{0}\right)$, and $\operatorname{Runs}(A)=\operatorname{Runs}^{*}(A) \cup \operatorname{Runs}^{\omega}(A)$. If $\varrho$ is finite and ends in $s_{n}$, we let last $(\varrho)=s_{n}$. Because of the denseness of the time domain, the unfolding of $A$ as a graph is infinite (uncountable number of states and delay edges). The trace, $\operatorname{tr}(\varrho)$, of a run $\varrho$ is the timed word $\boldsymbol{\pi}_{\Sigma}\left(\delta_{0} a_{0} \delta_{1} a_{1} \cdots a_{n} \delta_{n} \cdots\right)$. The duration of the run $\varrho$ is $\operatorname{Duration}(\varrho)=\operatorname{Duration}(\operatorname{tr}(\varrho))$. For $V \subseteq \operatorname{Runs}(A)$, we let $\operatorname{Tr}(V)=\{\operatorname{tr}(\varrho) \mid \varrho \in V\}$, which is the set of traces of the runs in $V$.

A finite (resp. infinite) timed word $w$ is accepted by $A$ if it is the trace of a run of $A$ that ends in an $F$-location (resp. a run that reaches infinitely often an $R$-location). $\mathcal{L}^{*}(A)$ (resp. $\left.\mathcal{L}^{\omega}(A)\right)$ is the set of traces of finite (resp. infinite) timed words accepted by $A$, and $\mathcal{L}(A)=\mathcal{L}^{*}(A) \cup \mathcal{L}^{\omega}(A)$ is the set of timed words accepted by $A$.

In the sequel we often omit the sets $R$ and $F$ in TA and this implicitly means $F=L$ and $R=\varnothing$.

A timed automaton $A$ is deterministic if there is no $\tau$ labelled transition in $A$, and if, whenever $\left(\ell, g, a, r, \ell^{\prime}\right)$ and $\left(\ell, g^{\prime}, a, r^{\prime}, \ell^{\prime \prime}\right)$ are transitions of $A, g \wedge g^{\prime} \equiv$ FALSE. $A$ is complete if from each state $(\ell, v)$, and for each action $a$, there is a transition $\left(\ell, g, a, r, \ell^{\prime}\right)$ such that $v \models g$. We note DTA the class of deterministic timed automata.

A finite automaton is a particular TA with $X=\varnothing$. Consequently guards and invariants are vacuously true and time elapsing transitions do not exist. We write $A=\left(Q, q_{0}, \Sigma_{\tau}, E, F, R\right)$ for a finite automaton. A run is thus a sequence of the form:

$$
\varrho=\ell_{0} \stackrel{a_{0}}{\longrightarrow} \ell_{1} \ldots \ldots \stackrel{a_{n-1}}{\longrightarrow} \ell_{n} \ldots
$$

where for each $i \geq 0,\left(\ell_{i}, a_{i}, \ell_{i+1}\right) \in E$. Definitions of traces and languages are the same as for TA. For FA, the duration of a run $\varrho$ is the number of steps (including $\tau$-steps) of $\varrho$ : if $\varrho$ is finite and ends in $\ell_{n}$, Duration $(\varrho)=n$ and otherwise $\operatorname{Duration}(\varrho)=\infty$.

\subsection{Region Graph of a Timed Automaton}

A region of $\mathbb{R}_{>0}^{X}$ is a conjunction of atomic constraints of the form $x \bowtie c$ or $x-y \bowtie c$ with $c \in \mathbb{Z}, \bowtie \in\{\leq,<,=,>, \geq\}$ and $x, y \in X$. The region graph $R G(A)$ of a TA $A$ is a finite quotient of the infinite graph of $A$ which is timeabstract bisimilar to $A$ [2]. It is a finite automaton on the alphabet $E^{\prime}=E \cup\{\tau\}$. The states of $R G(A)$ are pairs $(\ell, r)$ where $\ell \in L$ is a location of $A$ and $r$ is a region of $\mathbb{R}_{>0}^{X}$. More generally, the edges of the graph are tuples $\left(s, t, s^{\prime}\right)$ where $s, s^{\prime}$ are states of $R G(A)$ and $t \in E^{\prime}$. Genuine unobservable moves of $A$ labelled $\tau$ are labelled by tuples of the form $\left(s,(g, \tau, r), s^{\prime}\right)$ in $R G(A)$. An edge $(g, \lambda, R)$ in the region graph corresponds to a discrete transition of $A$ with guard $g$, action $\lambda$ and reset set $R$. A $\tau$ move in $R G(A)$ stands for a delay move to the timesuccessor region. The initial state of $R G(A)$ is $\left(l_{0}, \overline{0}\right)$. A final (resp. repeated) 
state of $R G(A)$ is a state $(\ell, r)$ with $\ell \in F$ (resp. $\ell \in R$ ). A fundamental property of the region graph [2] is:

Theorem 1 (R. Alur and D. Dill, [2]). $\mathcal{L}(R G(A))=\operatorname{Unt}(\mathcal{L}(A))$.

In other words:

1. if $w$ is accepted by $R G(A)$, then there is a timed word $v$ with $\operatorname{Unt}(v)=w$ s.t. $v$ is accepted by $A$.

2. if $v$ is accepted by $A$, then $\operatorname{Unt}(w)$ is accepted $R G(A)$.

The (maximum) size of the region graph is exponential in the number of clocks and in the maximum constant of the automaton $A$ (see [2]): $|R G(A)|=|L| \cdot|X|$ ! $2^{|X|} \cdot K^{|X|}$ where $K$ is the largest constant used in $A$.

\subsection{Product of Timed Automata}

Given a $n$ locations $\ell_{1}, \cdots, \ell_{n}$, we write $\bar{\ell}$ for the tuple $\left(\ell_{1}, \cdots, \ell_{n}\right)$ and let $\bar{\ell}[i]=\ell_{i}$. Given a letter $a \in \Sigma^{1} \cup \cdots \cup \Sigma^{n}$, we let $I(a)=\left\{k \mid a \in \Sigma^{k}\right\}$.

Definition 2 (Product of TA). Let $A_{i}=\left(L_{i}, l_{0}^{i}, X_{i}, \Sigma_{\tau}^{i}, E_{i}, I_{n} v_{i}\right), i \in$ $\{1, \cdots, n\}$, be $n$ TA s.t. $X_{i} \cap X_{j}=\varnothing$ for $i \neq j$. The product of the $A_{i}$ is the TA $A=A_{1} \times \cdots \times A_{n}=\left(L, l_{0}, X, \Sigma_{\tau}, E\right.$, Inv $)$ given by:

$-L=L_{1} \times \cdots \times L_{n}$

$-\overline{l_{0}}=\left(l_{0}^{1}, \cdots, l_{0}^{n}\right)$;

$-\Sigma=\Sigma^{1} \cup \cdots \cup \Sigma^{n}$

$-X=X_{1} \cup \cdots \cup X_{n}$

- $E \subseteq L \times \mathcal{C}(X) \times \Sigma_{\tau} \times 2^{X} \times L$ and $\left(\bar{\ell}, g, a, r, \bar{\ell}^{\prime}\right) \in E$ if:

- either $a \in \Sigma \backslash\{\tau\}$, and

1. for each $k \in I(a),\left(\bar{\ell}[k], g_{k}, a, r_{k}, \bar{\ell}^{\prime}[k]\right) \in E_{k}$,

2. $g=\wedge_{k \in I(a)} g_{k}$ and $r=\cup_{k \in I(a)} r_{k}$;

3. for $k \notin I(a), \bar{\ell}^{\prime}[k]=\bar{\ell}[k]$;

- or $a=\tau$ and $\exists j$ s.t. $\left(\bar{\ell}[j], g_{j}, \tau, r_{j}, \bar{\ell}^{\prime}[j]\right) \in E_{j}, g=g_{j}, r=r_{j}$ and for $k \neq j, \bar{\ell}^{\prime}[k]=\bar{\ell}[k]$.

$-\operatorname{Inv}(\bar{\ell})=\wedge_{k=1}^{n} \operatorname{Inv}(\bar{\ell}[k])$.

This definition of product also applies to finite automata (no clock constraints).

If the automaton $A_{i}$ has the set of final locations $F_{i}$ then the set of final locations for $A$ is $F_{1} \times \cdots \times F_{n}$. For Büchi acceptance, we add a counter $c$ to $A$ which is incremented every time the product automaton $A$ encounters an $R_{i}$-location in $A_{i}$, following the standard construction for product of Büchi automata. The automaton constructed with the counter $c$ is $A^{+}$. The repeated set of states of $A^{+}$is $L_{1} \times \cdots \times L_{n-1} \times L_{n} \times\{n\}$. As the sets of clocks of the $A_{i}$ 's are disjoint 2 , the following holds:

Fact $1 \mathcal{L}^{*}(A)=\cap_{i=1}^{n} \mathcal{L}^{*}\left(A_{i}\right)$ and $\mathcal{L}^{\omega}\left(A^{+}\right)=\cap_{i=1}^{n} \mathcal{L}^{\omega}\left(A_{i}\right)$.

\footnotetext{
${ }^{2}$ For finite automata, this is is vacuously true.
} 


\subsection{Intersection Emptiness Problem}

In this section we give some complexity results for the emptiness problem on products of FA and TA.

First consider the following problem on deterministic finite automata (DFA):

Problem 1 (Intersection Emptiness for DFA)

INPUTS: $n$ deterministic finite automata $A_{i}, 1 \leq i \leq n$, over the alphabet $\Sigma$. Problem: Check whether $\cap_{i=1}^{n} \mathcal{L}^{*}\left(A_{i}\right) \neq \varnothing$.

The size of the input for Problem 1 is $\sum_{i=1}^{n}\left|A_{i}\right|$.

Theorem 2 (D. Kozen, [14]). Problem 1] is PSPACE-complete.

D. Kozen's Theorem also holds for Büchi languages:

Theorem 3. Checking whether $\cap_{i=1}^{n} \mathcal{L}^{\omega}\left(A_{i}\right) \neq \varnothing$ is PSPACE-complete.

We establish a variant of Theorem 2 which will be used later in the paper: we show that Problem 1 is PSPACE-hard even if $A_{2}, \cdots, A_{n}$ are automata where all the states are accepting and $A_{1}$ is the only automaton with a proper set of accepting states (actually one accepting state is enough).

Proposition 1. Let $A_{i}, 1 \leq i \leq n$ be $n$ DTA over the alphabet $\Sigma$. If for all $A_{i}, 2 \leq i \leq n$, all states of $A_{i}$ are accepting, Problem 1 is already PSPACE-hard.

Proof. Let $A_{1}, A_{2}, \cdots, A_{n}$ be $n$ deterministic automata with accepting states $F_{1}, F_{2}, \cdots, F_{n}$ on the alphabet $\Sigma$. Let $\lambda$ be a fresh letter not in $\Sigma$. Define automaton $A_{i}^{\prime}$ by: from any state $q$ in $F_{i}$, add a transition $(q, \lambda, \perp)$ where $\perp$ is new state. Let $F_{1}^{\prime}=\{\perp\}$ and $F_{i}^{\prime}$ be all the states of $A_{i}^{\prime}$. It is clear that $\mathcal{L}^{*}\left(A_{1}^{\prime}\right)=\mathcal{L}^{*}\left(A_{1}\right) \cdot \lambda$.

We can prove that $\cap_{i=1}^{n} \mathcal{L}^{*}\left(A_{i}\right) \neq \varnothing \Longleftrightarrow \cap_{i=1}^{n} \mathcal{L}^{*}\left(A_{i}^{\prime}\right) \neq \varnothing$. Indeed, assume $w \in \cap_{i=1}^{n} \mathcal{L}^{*}\left(A_{i}\right) \neq \varnothing$. Then $A_{1} \times A_{2} \times \cdots \times A_{n}$ reaches the state $\left(q_{1}, q_{2}, \cdots, q_{n}\right)$ after reading $w$ and $\forall 1 \leq i \leq n, q_{i} \in F_{i}$. Thus in $A_{1}^{\prime} \times A_{2}^{\prime} \times \cdots \times A_{n}^{\prime}$ the same state can be reached and then $\lambda$ can be fired in the product leading to $(\perp, \perp, \cdots, \perp)$. Conversely, if a word $w$ is accepted by the product $A_{1}^{\prime} \times \cdots \times A_{n}^{\prime}, w$ must end with $\lambda$. Let $w=u . \lambda \in \cap_{i=1}^{n} \mathcal{L}^{*}\left(A_{i}^{\prime}\right) \neq \varnothing$. After reading $u$ the state of the product must be $\left(q_{1}, q_{2}, \cdots, q_{n}\right)$ with $\forall 1 \leq i \leq n, q_{i} \in F_{i}$, and the transitions fired when reading $u$ are also in $A_{1} \times A_{2} \times \cdots \times A_{n}$ which implies $u \in \cap_{i=1}^{n} \mathcal{L}^{*}\left(A_{i}\right)$.

The next results are counterparts of D. Kozen's results for TA.

Problem 2 (Intersection Emptiness for TA)

InPUTS: $n$ TA $A_{i}=\left(L_{i}, l_{0}^{i}, X_{i}, \Sigma_{\tau}^{i}, E_{i}\right.$, Inv $\left._{i}, F_{i}\right), 1 \leq i \leq n$ with $X_{k} \cap X_{j}=\varnothing$ for $k \neq j$.

PROBLem: Check whether $\cap_{i=1}^{n} \mathcal{L}^{*}\left(A_{i}\right) \neq \varnothing$.

Theorem 4. Problem 2 is PSPACE-complete. 
Proof. PSPACE-hardness follows from the fact that checking $\cap_{i=1}^{n} \mathcal{L}^{*}\left(A_{i}\right) \neq \varnothing$ on finite automata is already PSPACE-hard [14] or alternatively because reachability for timed automata is PSPACE-hard [2].

PSPACE-easiness can be established as Theorem 31 (section 4.1) of [1]: the regions of the product of TA $A_{i}$ can be encoded in polynomial space in the size of the clock constraints of the product automaton. An algorithm to check emptiness is obtained by: 1) guessing a sequence of pairs (location,region) in the product automaton and 2) checking whether it is accepted. This can be done in NPSPACE and by Savitch's Theorem in PSPACE.

The previous theorem extends to Büchi languages:

Problem 3 (Büchi Intersection Emptiness for TA)

InPUTS: $n$ TA $A_{i}=\left(L_{i}, l_{0}^{i}, X_{i}, \Sigma_{\tau}^{i}, E_{i}\right.$, Inv $\left._{i}, R_{i}\right), 1 \leq i \leq n$ with $X_{k} \cap X_{j}=\varnothing$ for $k \neq j$.

PRoBlem: Check whether $\cap_{i=1}^{n} \mathcal{L}^{\omega}\left(A_{i}\right) \neq \varnothing$.

Theorem 5. Problem 0 is PSPACE-complete.

Proof. PSPACE-hardness follows from the reduction of Problem 2 to Problem 3 or again because checking Büchi emptiness for timed automata is PSPACEhard [2.

Consider the product automaton $A^{+}$the construction of which is described at the end of section 2.5. PSPACE-easiness is established by: 1) guessing a state of $R G\left(A^{+}\right)$of the form $((\bar{\ell}, n), r)$ and 2$)$ checking it is reachable from the initial state (PSPACE) and reachable from itself (PSPACE). As $n$ is represented in binary the result follows.

\section{Fault Codiagnosis Problems}

We first recall the basics of fault diagnosis. The purpose of fault diagnosis [18] is to detect a fault in a system as soon as possible. The assumption is that the model of the system is known, but only a subset $\Sigma_{o}$ of the set of events $\Sigma$ generated by the system are observable. Faults are also unobservable.

Whenever the system generates a timed word $w \in T W^{*}(\Sigma)$, an external observer can only see $\boldsymbol{\pi}_{\Sigma_{o}}(w)$. If an observer can detect faults under this partial observation of the outputs of $A$, it is called a diagnoser. We require a diagnoser to detect a fault within a given delay $\Delta \in \mathbb{N}$.

To model timed systems with faults, we use timed automata on the alphabet $\Sigma_{\tau, f}=\Sigma_{\tau} \cup\{f\}$ where $f$ is the faulty (and unobservable) event. We only consider one type of fault, but the results we give are valid for many-types of faults $\left\{f_{1}, f_{2}, \cdots, f_{n}\right\}$ : indeed solving the many-types diagnosability problem amounts to solving $n$ one-type diagnosability problems [21]. The observable events are given by $\Sigma_{o} \subseteq \Sigma$ and $\tau$ is always unobservable.

The idea of decentralized or distributed diagnosis was introduced in [10]. It is based on decentralized architectures: local diagnosers and a communication 
protocol. In these architectures, local diagnosers (with their own partial view of the system) can send to a coordinator some information, using a given communication protocol. The coordinator then computes a result from the partial results of the local diagnosers. The goal is to obtain a coordinator that can detect the faults in the system. When local diagnosers do not communicate with each other nor with a coordinator (protocol 3 in [10]), the decentralized diagnosis problem is called codiagnosis [1520. In this section we formalize the notion of codiagnosability introduced in [15] in a style similar to 8]. This allows us to obtain a necessary and sufficient condition for codiagnosability of FA but also to extend the definition of codiagnosability to timed automata.

In the sequel we assume that the model of the system is a TA $A=\left(L, l_{0}, X\right.$, $\Sigma_{\tau, f}, E$, Inv) and is fixed.

\subsection{Faulty Runs}

Let $\Delta \in \mathbb{N}$. A run $\varrho$ of $A$ of the form

$$
\left(\ell_{0}, v_{0}\right) \stackrel{\delta_{0}}{\longrightarrow}\left(\ell_{0}, v_{0}+\delta_{0}\right) \stackrel{a_{0}}{\longrightarrow}\left(\ell_{1}, v_{1}\right) \quad \cdots \stackrel{a_{n-1}}{\longrightarrow}\left(\ell_{n}, v_{n}\right) \stackrel{\delta_{n}}{\longrightarrow}\left(\ell_{n}, v_{n}+\delta\right) \cdots
$$

is $\Delta$-faulty if: (1) there is an index $i$ s.t. $a_{i}=f$ and (2) the duration of $\varrho^{\prime}=$ $\left(\ell_{i}, v_{i}\right) \stackrel{\delta_{i}}{\longrightarrow} \cdots \stackrel{\delta_{n}}{\longrightarrow}\left(\ell_{n}, v_{n}+\delta_{n}\right) \cdots$ is larger than $\Delta$. We let Faulty ${ }_{\geq \Delta}(A)$ be the set of $\Delta$-faulty runs of $A$. Note that by definition, if $\Delta^{\prime} \geq \Delta$ then Faulty $\geq \Delta^{\prime}(A) \subseteq$ Faulty $_{>\Delta}(A)$. We let Faulty $(A)=\cup_{\Delta \geq 0}$ Faulty $_{\geq \Delta}(A)=$ Faulty $_{>0}(A)$ be the set of faulty runs of $A$, and $\operatorname{NonFaulty}(\bar{A})=\operatorname{Runs}(A) \backslash$ Faulty $(\bar{A})$ be the set of non-faulty runs of $A$. Finally, we let

$$
\text { Faulty }_{\geq \Delta}^{t r}(A)=\operatorname{Tr}\left(\text { Faulty }_{\geq \Delta}(A)\right)
$$

and

$$
\text { NonFaulty }^{\text {tr }}(A)=\operatorname{Tr}(\operatorname{NonFaulty}(A))
$$

which are the traces 3 of $\Delta$-faulty and non-faulty runs of $A$.

We also make the assumption that the TA $A$ cannot prevent time from elapsing. For FA, this assumption is that from any state, a discrete transition can be taken. If it is not case, $\tau$ loop actions can be added with no impact on the (co)diagnosability status of the system. This is a standard assumption in diagnosability and is required to avoid taking into account these cases that are not interesting in practice.

For discrete event systems (FA), the notion of time is the number of transitions (discrete steps) in the system. A $\Delta$-faulty run is thus a run with a fault action $f$ followed by at least $\Delta$ discrete steps (some of them can be $\tau$ or even $f$ actions). When we consider codiagnosability problems for discrete event systems, this definition of $\Delta$-faulty runs apply. The other definitions are unchanged.

\footnotetext{
$\overline{{ }^{3} \text { Notice that }} \operatorname{tr}(\varrho)$ erases $\tau$ and $f$.
} 
Remark 1. Using a timed automaton where discrete actions are separated by one time unit is not equivalent to using a finite automaton when solving a fault diagnosis problem. For instance, a timed automaton can generate the timed words

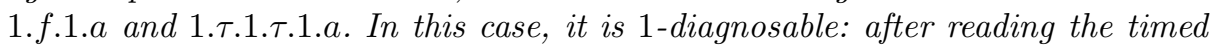
word 2.a we announce a fault. If we do not see the 1-time unit durations, the timed words f.a and $\tau^{2}$. a give the same observation. And thus it is not diagnosable if we cannot measure time. Using a timed automaton where discrete actions are separated by one time unit gives to the diagnoser the ability to count/measure time and this is not equivalent to the fault diagnosis problem for FA (discrete event systems).

\subsection{Codiagnosers and Codiagnosability Problems}

A codiagnoser is a tuple of diagnosers, each of which has its own set of observable events $\Sigma_{i}$, and whenever a fault occurs, at least one diagnoser is able to detect it. In the sequel we write $\boldsymbol{\pi}_{i}$ in place of $\boldsymbol{\pi}_{\Sigma_{i}}$ for readability reasons. A codiagnoser can be formally defined as follows:

Definition $3((\Delta, \mathcal{E})$-Codiagnoser $)$. Let $A$ be a timed automaton over the alphabet $\Sigma_{\tau, f}, \Delta \in \mathbb{N}$ and $\mathcal{E}=\left(\Sigma_{i}\right)_{1 \leq i \leq n}$ be a family of subsets of $\Sigma$. $A(\Delta, \mathcal{E})$ codiagnoser for $A$ is a mapping $\bar{D}=\left(D_{1}, \cdots, D_{n}\right)$ with $D_{i}: T W^{*}\left(\Sigma_{i}\right) \rightarrow\{0,1\}$ such that:

- for each $\varrho \in N o n F a u l t y(A), \sum_{i=1}^{n} \bar{D}[i]\left(\boldsymbol{\pi}_{i}(\operatorname{tr}(\varrho))\right)=0$,

- for each $\varrho \in$ Faulty $_{\geq \Delta}(A), \sum_{i=1}^{n} \bar{D}[i]\left(\boldsymbol{\pi}_{i}(\operatorname{tr}(\varrho))\right) \geq 1$.

As for diagnosability, the intuition of this definition is that $(i)$ the codiagnoser will raise an alarm $(\bar{D}$ outputs a value different from 0$)$ when a $\Delta$-faulty run has been identified, and that $(i i)$ it can identify those $\Delta$-faulty runs unambiguously. The codiagnoser is not required to do anything special for $\Delta^{\prime}$-faulty runs with $\Delta^{\prime}<\Delta$ (although it is usually required that once it has announced a fault, it does not change its mind and keep outputting 1 ).

$A$ is $(\Delta, \mathcal{E})$-codiagnosable if there exists a $(\Delta, \mathcal{E})$-codiagnoser for $A . A$ is $\mathcal{E}$-codiagnosable if there is some $\Delta \in \mathbb{N}$ s.t. $A$ is $(\Delta, \mathcal{E})$-codiagnosable.

The standard notions [18] of $\Delta$-diagnosability and $\Delta$-diagnoser are obtained when the family $\mathcal{E}$ is the singleton $\mathcal{E}=\{\Sigma\}$. The fundamental codiagnosability problems for timed automata are the following:

Problem $4((\Delta, \mathcal{E})$-Codiagnosability)

InPuts: $A$ TA $A=\left(L, l_{0}, X, \Sigma_{\tau, f}, E\right.$, Inv $), \Delta \in \mathbb{N}$ and $\mathcal{E}=\left(\Sigma_{i}\right)_{1 \leq i \leq n}$.

Problem: Is $A(\Delta, \mathcal{E})$-codiagnosable?

Problem 5 (Codiagnosability)

InPuts: $A$ TA $A=\left(L, l_{0}, X, \Sigma_{\tau, f}, E\right.$, Inv $)$ and $\mathcal{E}=\left(\Sigma_{i}\right)_{1 \leq i \leq n}$.

Problem: Is $A \mathcal{E}$-codiagnosable? 


\section{Problem 6 (Optimal delay)}

InPUTS: $A$ TA $A=\left(L, l_{0}, X, \Sigma_{\tau, f}, E\right.$, Inv $)$ and $\mathcal{E}=\left(\Sigma_{i}\right)_{1 \leq i \leq n}$.

Problem: If $A$ is $\mathcal{E}$-codiagnosable, what is the minimum $\Delta$ s.t. $A$ is $(\Delta, \mathcal{E})$ codiagnosable?

The size of the input for Problem 4 is $|A|+\log \Delta+n \cdot|\Sigma|$, and for Problems 5 and 6 it is $|A|+n \cdot|\Sigma|$.

In addition to the previous problems, we will consider the construction of a $(\Delta, \mathcal{E})$-codiagnoser when $A$ is $(\Delta, \mathcal{E})$-codiagnosable in section 5

\subsection{Necessary and Sufficient Condition for Codiagnosability}

In this section we generalize the necessary and sufficient condition for diagnosability [19]8] to codiagnosability.

Lemma 1. $A$ is not $(\Delta, \mathcal{E})$-codiagnosable if and only if $\exists \varrho \in$ Faulty $_{\geq \Delta}(A)$ and

$$
\forall 1 \leq i \leq n, \exists \varrho_{i} \in \operatorname{NonFaulty}(A) \text { s.t. } \boldsymbol{\pi}_{i}(\operatorname{tr}(\varrho))=\boldsymbol{\pi}_{i}\left(\operatorname{tr}\left(\varrho_{i}\right)\right) .
$$

Proof.

- Only if part. Assume equation (1) holds and $A$ is $(\Delta, \mathcal{E})$-codiagnosable. Then there is a codiagnoser $\bar{D}=\left(D_{1}, \cdots, D_{n}\right)$ satisfying Definition 3. For each $\varrho_{i}$ we must have $D_{i}\left(\boldsymbol{\pi}_{i}\left(\operatorname{tr}\left(\varrho_{i}\right)\right)\right)=0$ because each $\varrho_{i}$ is non faulty. But we must also have for at least one index $i, D_{i}\left(\boldsymbol{\pi}_{i}\left(\operatorname{tr}\left(\varrho_{i}\right)\right)\right)=D_{i}\left(\boldsymbol{\pi}_{i}(\operatorname{tr}(\varrho))\right)=1$ because $\varrho$ is $\Delta$-faulty, which is impossible.

- If part. Assume $A$ is not $(\Delta, \mathcal{E})$-codiagnosable and $\forall \varrho \in$ Faulty $_{\geq \Delta}(A)$, equation (11) does not hold. In this case, there is an index $1 \leq i \leq n$ s.t. :

$$
\forall \varrho^{\prime} \in \operatorname{NonFaulty}(A), \quad \boldsymbol{\pi}_{i}(\operatorname{tr}(\varrho)) \neq \boldsymbol{\pi}_{i}\left(\operatorname{tr}\left(\varrho^{\prime}\right)\right) .
$$

Define $D_{i}(w)=1$ when $w \in \boldsymbol{\pi}_{i}\left(\right.$ Faulty $\left._{\geq \Delta}^{\text {tr }}(A)\right) \backslash \boldsymbol{\pi}_{i}\left(\right.$ NonFaulty $\left.^{\text {tr }}(A)\right)$ and 0 otherwise. Then $\bar{D}=\left(D_{1}, \cdots, D_{n}\right)$ is a $\Delta$-codiagnoser for $A$. Indeed, let $\varrho \in \operatorname{NonFaulty}(A)$. Then $\boldsymbol{\pi}_{i}(\operatorname{tr}(\varrho)) \in \boldsymbol{\pi}_{i}\left(\operatorname{NonFaulty}^{\operatorname{tr}}(A)\right)$ and this implies that $D_{i}\left(\boldsymbol{\pi}_{i}(\operatorname{tr}(\varrho))\right)=0$. Let $\varrho \in$ Faulty $y_{\geq \Delta}(A)$ and assume $D_{i}\left(\boldsymbol{\pi}_{i}(\operatorname{tr}(\varrho))\right)=$ 0 for each $1 \leq i \leq n$. By definition of $D_{i}$ we must have $\pi_{i}(\operatorname{tr}(\varrho)) \in$ $\boldsymbol{\pi}_{i}\left(\operatorname{NonFaulty}^{t r}(A)\right)$. In this case, there is some run $\varrho_{i} \in \operatorname{NonFaulty}(A)$ s.t. $\boldsymbol{\pi}_{i}(\operatorname{tr}(\varrho))=\boldsymbol{\pi}_{i}\left(\operatorname{tr}\left(\varrho_{i}\right)\right)$ and thus equation (1) holds which contradicts the initial assumption.

Using Lemma 1, we obtain a language based characterisation of codiagnosability extending the one given in [19|8]. Let $\boldsymbol{\pi}_{i}^{-1}(X)=\left\{w \in T W^{*}(\Sigma) \mid \boldsymbol{\pi}_{i}(w) \in X\right\}$.

Lemma 2. $A$ is $(\Delta, \mathcal{E})$-codiagnosable if and only if

$$
\text { Faulty }_{\geq \Delta}^{t r}(A) \cap\left(\bigcap_{i=1}^{n} \pi_{i}^{-1}\left(\boldsymbol{\pi}_{i}\left(\operatorname{NonFaulty}^{\text {tr }}(A)\right)\right)\right)=\varnothing .
$$


Proof. Assume equation 2 does not hold and let $w \in$ Faulty $_{\geq \Delta}^{t r}(A)$, and for each $1 \leq i \leq n, w \in \boldsymbol{\pi}_{i}^{-1}\left(\boldsymbol{\pi}_{i}\left(\right.\right.$ NonFaulty $\left.\left.^{t r}(A)\right)\right)$. This implies that:

$-\exists \varrho \in$ Faulty $_{\geq \Delta}(A)$ s.t. $\operatorname{tr}(\varrho)=w$;

- for each $i, w \in \boldsymbol{\pi}_{i}^{-1}\left(\boldsymbol{\pi}_{i}\left(\right.\right.$ NonFaulty $\left.\left.^{t r}(A)\right)\right)$ and $\boldsymbol{\pi}_{i}(w) \in \boldsymbol{\pi}_{i}\left(\right.$ NonFaulty $\left.^{\text {tr }}(A)\right)$.

Thus, there is a run $\varrho_{i} \in \operatorname{NonFaulty}(A)$, s.t. $\boldsymbol{\pi}_{i}(w)=\boldsymbol{\pi}_{i}(\operatorname{tr}(\varrho))=\boldsymbol{\pi}_{i}\left(\operatorname{tr}\left(\varrho_{i}\right)\right)$ and as equation (1) of Lemma 1 is satisfied, $A$ is not $(\Delta, \mathcal{E})$-codiagnosable.

For the converse, assume $A$ is not $(\Delta, \mathcal{E})$-codiagnosable. By Lemma 1 , equation (11) is satisfied and:

- there is a run $\varrho$ with $\operatorname{tr}(\varrho) \in$ Fault $_{>}^{\text {tr }}>\Delta(A)$;

- for each $i$, there is some $\varrho_{i} \in \operatorname{NonFaulty}(A)$ s.t. $\boldsymbol{\pi}_{i}(\operatorname{tr}(\varrho))=\boldsymbol{\pi}_{i}\left(\operatorname{tr}\left(\varrho_{i}\right)\right)$. Hence $\operatorname{tr}(\varrho) \in \boldsymbol{\pi}_{i}^{-1}\left(\boldsymbol{\pi}_{i}\left(\right.\right.$ NonFaulty $\left.\left.^{\operatorname{tr}}(A)\right)\right)$ for each $i$,

and this implies that equation 2 does not hold.

\section{Algorithms for Codiagnosability Problems}

\section{$4.1(\Delta, \mathcal{E})$-Codiagnosability (Problem 4 )}

Deciding Problem 4 amounts to checking whether equation 2 holds or not. Recall that $A=\left(L, l_{0}, X, \Sigma_{\tau, f}, E\right.$, Inv $)$. Let $t$ be a fresh clock not in $X$. Let $A^{f}(\Delta)=$ $\left((L \times\{0,1\}) \cup\{B a d\},\left(l_{0}, 0\right), X \cup\{t\}, \Sigma_{\tau}, E_{f}, I n v_{f}\right)$ with:

- $\left((\ell, n), g, \lambda, r,\left(\ell^{\prime}, n\right)\right) \in E_{f}$ if $\left(\ell, g, \lambda, r, \ell^{\prime}\right) \in E, \lambda \in \Sigma \cup\{\tau\}$;

- $\left((\ell, 0), g, \tau, r \cup\{t\},\left(\ell^{\prime}, 1\right)\right) \in E_{f}$ if $\left(\ell, g, f, r, \ell^{\prime}\right) \in E$;

- for $\ell \in L,((\ell, 1), t \geq \Delta, \tau, \varnothing, B a d) \in E_{f}$;

$-\operatorname{Inv}_{f}((\ell, n))=\operatorname{Inv}(\ell)$.

$A^{f}(\Delta)$ is similar to $A$ but when a fault occurs it switches to a copy of $A$ (encoded by $n=1$ ). When sufficient time has elapsed in the copy (more than $\Delta$ time units), location $B a d$ can be reached.

The language accepted by $A^{f}(\Delta)$ with the set of final states $\{B a d\}$ is thus $\mathcal{L}^{*}\left(A^{f}(\Delta)\right)=$ Faulty $_{\geq \Delta}^{\text {tr }}(A)$. Define $A_{i}=\left(L, l_{0}, X_{i}, \Sigma_{\tau}, E_{i}\right.$, Inv $\left._{i}\right)$ with:

- $X_{i}=\left\{x^{i} \mid x \in X\right\}$ (create copies of clocks of $A$ );

- $\left(\ell, g_{i}, \lambda, r_{i}, \ell^{\prime}\right) \in E_{i}$ if $\left(\ell, g, \lambda, r, \ell^{\prime}\right) \in E, \lambda \in \Sigma_{i} \cup\{\tau\}$ with: $g_{i}$ is $g$ where the clocks $x$ in $X$ are replaced by their counterparts $x^{i}$ in $X_{i} ; r_{i}$ is $r$ with the same renaming;

- $\left(\ell, g_{i}, \tau, r_{i}, \ell^{\prime}\right) \in E_{i}$ if $\left(\ell, g, \lambda, r, \ell^{\prime}\right) \in E, \lambda \in \Sigma \backslash \Sigma_{i}$

- Invi $(\ell)=\operatorname{Inv}(\ell)$ with clock renaming $\left(x^{i}\right.$ in place of $\left.x\right)$.

Each $A_{i}$ accepts only non-faulty traces as the $f$-transitions are not in $A_{i}$. If the set of final locations is $L$ for each $A_{i}, \mathcal{L}^{*}\left(A_{i}\right)=\boldsymbol{\pi}_{i}\left(\right.$ NonFaulty $\left.^{t r}(A)\right)$. To accept $\boldsymbol{\pi}_{i}^{-1}\left(\boldsymbol{\pi}_{i}\left(\operatorname{NonFaulty}^{\operatorname{tr}}(A)\right)\right.$ we add transitions $(\ell$, TRUE, $\lambda, \varnothing, \ell)$ for each location $\ell$ of $E_{i}$ and for each $\lambda \in \Sigma \backslash \Sigma_{i}$. Let $A_{i}^{*}$ be the automaton on the alphabet $\Sigma$ constructed this way. By definition of $A_{i}^{*}, \mathcal{L}^{*}\left(A_{i}^{*}\right)=\boldsymbol{\pi}_{i}^{-1}\left(\boldsymbol{\pi}_{i}\left(\operatorname{NonFaulty}^{t r}(A)\right)\right)$.

Define $\mathcal{B}=A^{f}(\Delta) \times A_{1}^{*} \times A_{2}^{*} \times \cdots \times A_{n}^{*}$ with the set of final locations $F_{\mathcal{B}}=\{B a d\} \times L \times \cdots \times L$. We let $R_{\mathcal{B}}=\varnothing$. Using equation 2 we obtain: 
Lemma 3. $A$ is $(\Delta, \mathcal{E})$-codiagnosable iff $\mathcal{L}^{*}(\mathcal{B})=\varnothing$.

Proof. The sets of clocks of the $A_{i}$ 's and $A^{f}(\Delta)$ are disjoint: for each $1 \leq i<$ $j \leq n, X_{i} \cap X_{j}=\varnothing$ and $X_{i} \cap X=\varnothing$. It follows from Fact 1 that $\mathcal{L}^{*}(\mathcal{B})=$ $\mathcal{L}^{*}\left(A^{f}(\Delta)\right) \cap\left(\bigcap_{i=1}^{n} \mathcal{L}^{*}\left(A_{i}^{*}\right)\right)$. By Lemma 2 and the construction of $A^{f}(\Delta)$ and the $A_{i}$ 's, the result follows.

The size of the input for problem 4 is $|A|+\log \Delta+n \cdot|\Sigma|$. The size of $A^{f}(\Delta)$ is (linear in) the size of $A$ and $\log \Delta$, i.e., $O(|A|+\log \Delta)$. The size of $A_{i}^{*}$ is also bounded by the size of $A$. If follows that $\left|A^{f}(\Delta)\right|+\sum_{i=1}^{n}\left|A_{i}^{*}\right|$ is bounded by $(n+1)|A|$ and is polynomial in the size of the input of problem 4 . We thus have a polynomial reduction from Problem 4 to the intersection emptiness problem for TA. We can now establish the following result:

Theorem 6. Problem 4 is PSPACE-complete for Timed Automata. It is already PSPACE-hard for Deterministic Finite Automata.

Proof. PSPACE-easiness follows from the polynomial reduction described above and Lemma 3. PSPACE-hardness is obtained by reducing the variant of the intersection emptiness problem for DTA to the $(\Delta, \mathcal{E})$-codiagnosability problem. This problem is PSPACE-hard (Proposition 1).

Let $A_{i}, 1 \leq i \leq n$, be $n$ deterministic finite automata over the alphabet $\Sigma$. Assume $A_{1}$ has one accepting state and for $A_{2}, \cdots, A_{n}$ all states are accepting.

We construct $B$ as shown on Figure 1 $a_{2}, \cdots, a_{n}$ are fresh letters not in $\Sigma$; the target state of $a_{i}$ is the initial state of $A_{i}$. The initial state of $B$ is $\iota$. Let $\Sigma_{i}=\Sigma \backslash\left\{a_{i}\right\}$ for each $2 \leq i \leq n$. From the final state of $A_{1}$ there is a transition labeled $f$ to a new state $e$.

We can prove that $B$ is $(1, \mathcal{E})$-diagnosable if and only if $\cap_{i=1}^{n} \mathcal{L}^{*}\left(A_{i}\right)=\varnothing$ with $\mathcal{E}=\left(\Sigma_{i}\right)_{1<i<n}$. Assume $w \in \cap_{i=1}^{n} \mathcal{L}^{*}\left(A_{i}\right) \neq \varnothing$. Take the run of trace $\tau . w . f . \tau$ in $B$. This run is 1-faulty. For each $2 \leq i \leq n$, there is a run of trace $a_{i} \cdot w$ which is non faulty. Moreover, $\boldsymbol{\pi}_{i}\left(a_{i} \cdot w\right)=w$ and thus $B$ is not $(1, \mathcal{E})$-codiagnosable.

Now, assume $B$ is not $(1, \mathcal{E})$-codiagnosable. There is a 1 -faulty run, and this must be a run of trace $\tau$.w.f. $\tau$ with $w \in \mathcal{L}^{*}\left(A_{1}\right)$, and for each $2 \leq i \leq n$, there is a non-faulty run $\varrho_{i}$ the trace of which is $u_{i}$, with $\boldsymbol{\pi}_{i}\left(u_{i}\right)=w$. It must be the case that $u_{i}=a_{i} \cdot w_{i}$ as otherwise $\boldsymbol{\pi}_{i}\left(u_{i}\right)$ would start with $a_{k}, k \neq i$ and thus it would be impossible to have $\boldsymbol{\pi}_{i}\left(u_{i}\right)=w$. As $u_{i}=a_{i} \cdot w_{i}, \boldsymbol{\pi}_{i}\left(u_{i}\right)=w_{i}=w$, and $w \in \mathcal{L}^{*}\left(A_{i}\right)$, it follows that $w \in \cap_{i=1}^{n} \mathcal{L}^{*}\left(A_{i}\right)$ and thus $\cap_{i=1}^{n} \mathcal{L}^{*}\left(A_{i}\right)$ is not empty. Finally $\cap_{i=1}^{n} \mathcal{L}^{*}\left(A_{i}\right) \neq \varnothing$ if and only if $B$ is not $(1, \mathcal{E})$-codiagnosable.

The size of $B$ is in $O\left(\sum_{i=1}^{n}\left|A_{i}\right|+n\right)$ which is equal to $O\left(\sum_{i=1}^{n}\left|A_{i}\right|\right)$ as $\left|A_{i}\right| \geq 1$. The size of the input for Problem 4 is thus $\left.O\left(\sum_{i=1}^{n}\left|A_{i}\right|\right)+n \cdot(|\Sigma|+n)\right)$ which is quadratic and thus polynomial in $\sum_{i=1}^{n}\left|A_{i}\right|$.

The intersection emptiness problem for DTA is polynomially reducible to the $(\Delta, \mathcal{E})$-codiagnosability Problem and Problem 4 is PSPACE-hard for DTA. 


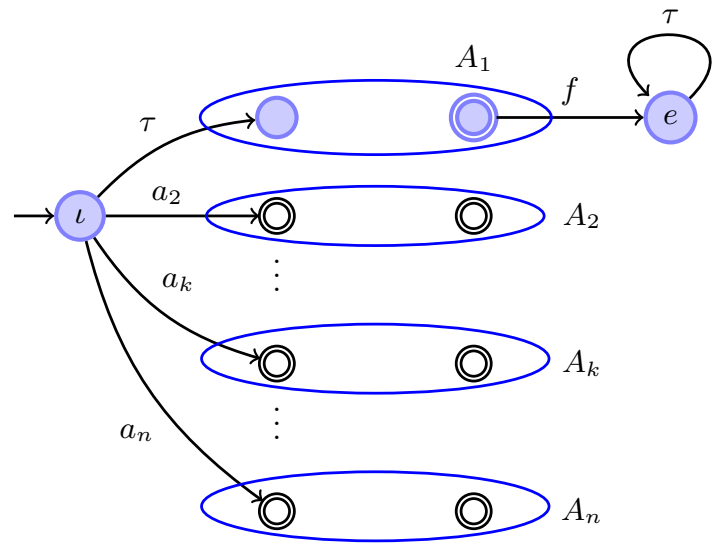

Fig. 1. Reduction for Theorem 4, Automaton $B$

\section{$4.2 \quad \mathcal{E}$-Codiagnosability (Problem 5 )}

In this section we show how to solve the $\mathcal{E}$-codiagnosability problem. The algorithm is a generalisation of the procedure for deciding diagnosability of discrete event and timed systems (see [7] for a recent presentation).

First notice that $A$ is not $\mathcal{E}$-diagnosable if and only if for all $\Delta \in \mathbb{N}, A$ is not $(\Delta, \mathcal{E})$-diagnosable. For standard fault diagnosis (one diagnoser and $\mathcal{E}=\{\Sigma\}$ ), $A$ is not diagnosable if there is an infinite faulty run in $A$ the projection of which is the same as the projection of a non-faulty one [7.

The procedure for checking diagnosability of FA and TA slightly differ due to specific features of timed systems. We recall here the algorithms to check diagnosability of FA and TA [7]19] and extend them to codiagnosability.

Codiagnosability for Finite Automata. To check whether a FA $A$ is diagnosable, we build a synchronized product $A^{f} \times A_{1}$, s.t. $A^{f}$ behaves exactly like $A$ but records in its state whether a fault has occurred, and $A_{1}$ behaves like $A$ without the faulty runs (transitions labelled $f$ are cut off). This corresponds to $A^{f}(\Delta)$ defined in section 4.1 without the clock $\Delta$.

A faulty run in the product $A^{f} \times A_{1}$ is a run for which $A^{f}$ reaches a faulty state of the form $(q, 1)$. To decide whether $A$ is diagnosable we build an extended version of $A^{f} \times A_{1}$ which is a Büchi automaton $\mathcal{B}$ [7]: $\mathcal{B}$ has a boolean variable $z$ which records whether $A^{f}$ participated in the last transition fired by $A^{f} \times A_{1}$. A state of $\mathcal{B}$ is a pair $(s, z)$ where $s$ is a state of $A^{f} \times A_{1}$. $\mathcal{B}$ is given by the tuple $\left((Q \times\{0,1\} \times Q) \times\{0,1\},\left(\left(q_{0}, 0\right), q_{0}, 0\right), \Sigma_{\tau}, \longrightarrow_{\mathcal{B}}, \varnothing, R_{\mathcal{B}}\right)$ with:

- $(s, z) \stackrel{\lambda}{\longrightarrow}\left(s^{\prime}, z^{\prime}\right)$ if $(i)$ there exists a transition $t: s \stackrel{\lambda}{\longrightarrow} s^{\prime}$ in $A^{f} \times A_{1}$, and (ii) $z^{\prime}=1$ if $\lambda$ is a move of $A^{f}$ and $z^{\prime}=0$ otherwise;

- $R_{\mathcal{B}}=\left\{\left(\left((q, 1), q^{\prime}\right), 1\right) \mid\left((q, 1), q^{\prime}\right) \in A^{f} \times A_{1}\right\}$. 
The important part of the previous construction relies on the fact that, for $A$ to be non $\Sigma$-diagnosable, $A^{f}$ should have an infinite faulty run (and take infinitely many transitions) and $A_{1}$ a corresponding non-faulty run (note that this one can be finite) giving the same observation. With the previous construction, we have [7]: $A$ is diagnosable iff $\mathcal{L}^{\omega}(\mathcal{B})=\varnothing$.

The construction for codiagnosability is an extension of the previous one adding $A_{2}, \cdots, A_{n}$ to the product. Let $\mathcal{B}^{c o}=A^{f} \times A_{1} \times \cdots \times A_{n}$ with $A_{i}$ defined in section 4.1. In $\mathcal{B}^{c o}$ we again use the variable $z$ to indicate whether $A^{f}$ participated in the last move. Define the set of repeated states of $\mathcal{B}^{c o}$ by: $R_{\mathcal{B}^{c o}}=\left\{(((q, 1), \bar{q}), 1) \mid((q, 1), \bar{q}) \in A^{f} \times A_{1} \times \cdots \times A_{n}\right\}$. By construction, a state in $R_{\mathcal{B}^{c o}}$ is: (1) faulty as it contains a component $(q, 1)$ for the state of $A^{f}$ and (2) $A^{f}$ participated in the last move as $z=1$. It follows that:

Lemma 4. $A$ is $\mathcal{E}$-codiagnosable iff $\mathcal{L}^{\omega}\left(\mathcal{B}^{c o}\right)=\varnothing$.

Theorem 7. Problem 5 is PSPACE-complete for DFA.

Proof. PSPACE-easiness follows form the fact that checking whether $\mathcal{L}^{\omega}\left(\mathcal{B}^{c o}\right)=$ $\varnothing$ can be done in PSPACE (Theorem 3). PSPACE-hardness follows from a reduction of Problem 1 to Problem 5 using the same encoding as the one given in the proof of Theorem 6 , the automaton $B$ of Fig. 1 is not $(\Delta, \mathcal{E})$-codiagnosable for any $\Delta \in \mathbb{N}$.

Codiagnosability for Timed Automata. Checking diagnosability for timed automata requires an extra step in the construction of the equivalent of automaton $\mathcal{B}$ defined above: indeed, for TA, a run having infinitely many discrete steps could well be zeno, i.e., the duration of such a run can be finite. This extra step in the construction was first presented in 19 . It can be carried out by adding a special timed automaton $\operatorname{Div}(x)$ and synchronizing it with $A^{f} \times A_{1}$. Let $x$ be a fresh clock not in $X$. Let $\operatorname{Div}(x)=(\{0,1\}, 0,\{x\}, E, \operatorname{Inv})$ be the TA given in Fig. 2. If we use $F=\varnothing$ and $R=\{1\}$ for $\operatorname{Div}(x)$, any accepted run is time

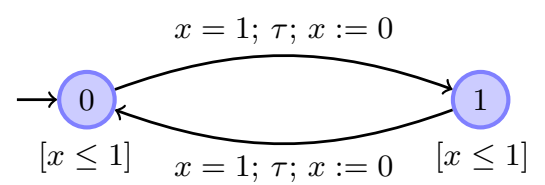

Fig. 2. Timed Automaton Div $(x)$

divergent and thus cannot be zeno. Let $\mathcal{D}=A^{f} \times \operatorname{Div}(x) \times A_{1}$ and let $F_{\mathcal{D}}=\varnothing$ and $R_{\mathcal{D}}$ be the set of states where $A^{f}$ is in a faulty location and $\operatorname{Div}(x)$ is in location 1. For standard fault diagnosis, the following holds [197]: $A$ is diagnosable iff $\mathcal{L}^{\omega}(\mathcal{D})=\varnothing$.

The construction to check codiagnosability is obtained by adding $A_{2}, \cdots, A_{n}$ in the product. Let $\mathcal{D}^{c o}=A^{f} \times \operatorname{Div}(x) \times A_{1} \times \cdots \times A_{n}$. 
Lemma 5. $A$ is $\mathcal{E}$-codiagnosable iff $\mathcal{L}^{\omega}\left(\mathcal{D}^{c o}\right)=\varnothing$.

Theorem 8. Problem 5 is PSPACE-complete for TA.

Proof. The size of $\mathcal{D}^{c o}$ is in $O((n+1) \cdot|A|)$ and thus polynomial in the size of the input of Problem $5(|A|+n \cdot|\Sigma|)$. PSPACE-easiness follows because the intersection emptiness problem for Büchi automata can be solved in PSPACE. PSPACE-hardness holds because it is already PSPACE-hard for FA.

\subsection{Optimal Delay (Problem 6)}

Using the results for checking $\mathcal{E}$-codiagnosability and $(\Delta, \mathcal{E})$-codiagnosability, we obtain algorithms for computing the optimal delay.

Lemma 4 reduces codiagnosability of FA to Büchi emptiness on a product automaton. The number of states of the automaton $\mathcal{B}^{\text {co }}$ is bounded by $4 \cdot|A|^{n}$, and the number of faulty states by $2 \cdot|A|^{n}$. This implies that:

Proposition 2. Let $A$ be a finite automaton. If $A$ is $\mathcal{E}$-codiagnosable, then $A$ is $\left(2 \cdot|A|^{n}, \mathcal{E}\right)$-codiagnosable.

Proof. If $\mathcal{L}\left(\mathcal{B}^{\text {co }}\right)=\varnothing$ there cannot be a faulty run of length more than $2 \cdot|A|^{n}$ otherwise at least one faulty state $s$ will be encountered twice on this run, and in this case we could construct an infinite faulty run which contradicts the fact that $\mathcal{L}\left(\mathcal{B}^{c o}\right)=\varnothing$.

From Proposition 2, we can conclude that:

Theorem 9. Problem [6 can be solved in PSPACE for FA.

Proof. Checking whether $A$ is $\mathcal{E}$-codiagnosable can be done in PSPACE. If the result is "yes", we can do a binary search for the optimal delay: start with $\Delta=2 \cdot|A|^{n}$, and check whether $A$ is $(\Delta, \mathcal{E})$-codiagnosable. If "yes", divide $\Delta$ by 2 and so on. The encoding of $2 \cdot|A|^{n}$ has size $O(n \cdot \log |A|)$ and thus is polynomial in the size of the inputs of Problem [6]

For timed automata, a similar reasoning can be done on the region graph of $\mathcal{D}^{c o}$. If a TA $A$ is $\mathcal{E}$-codiagnosable, there cannot be any cycle with faulty locations in $R G\left(\mathcal{D}^{c o}\right)$. Otherwise there would be a non-zeno infinite word in $\mathcal{L}\left(\mathcal{D}^{c o}\right)$ and thus an infinite time-diverging faulty run in $A$, with corresponding non-faulty runs in each $A_{i}$, giving the same observation. Let $K$ be the size of $R G\left(\mathcal{D}^{c o}\right)$. If $A$ is $\mathcal{E}$-codiagnosable, then a faulty state in $R G\left(\mathcal{D}^{c o}\right)$ can be followed by at most $K$ states. Otherwise a cycle in the region graph would occur and thus $\mathcal{L}^{\omega}\left(\mathcal{D}^{c o}\right)$ would not be empty. This also implies that all the states $(s, r)$ in $R G\left(\mathcal{D}^{c o}\right)$ that can follow a faulty state must have a bounded region. As the amount of time that can elapse in one region is at most 1 time unit 4 , the maximum duration of a faulty run in $\mathcal{D}^{c o}$ is bounded by $K$. This implies that:

\footnotetext{
${ }^{4}$ The constants in the automata are integers.
} 
Proposition 3. Let $A$ be a timed automaton. If $A$ is $\mathcal{E}$-codiagnosable, then $A$ is $(K, \mathcal{E})$-codiagnosable with $K=\left|R G\left(\mathcal{D}^{c o}\right)\right|$.

The size of the region graph of $\mathcal{D}^{c o}$ is bounded by $|L|^{n+1} \cdot((n+1)|X|+1)$ ! . $2^{(n+1)|X|+1} \cdot M^{(n+1)|X|+1}$. Thus the encoding of constant $K$ has size $O(n \cdot|A|)$.

Theorem 10. Problem [ [ can be solved in PSPACE for Timed Automata.

Proof. Checking whether a TA $A$ is $\mathcal{E}$-codiagnosable can be done in PSPACE. If the result is "yes", we can do a binary search for the maximum delay: start with $\Delta=K=\left|R G\left(\mathcal{B}^{c o}\right)\right|$, and check whether $A$ is $(\Delta, \mathcal{E})$-codiagnosable. If "yes", divide $\Delta$ by 2 and so on. The encoding of $K$ has size $O(n \cdot|A|)$ and thus is polynomial in the size of the input of Problem 6.

\section{Synthesis of Codiagnosers}

\subsection{Synthesis for Finite Automata}

The synthesis of a codiagnoser for a FA $A$ can be achieved by determinizing $n$ versions of $A$. This is exactly the same procedure that is applied for standard diagnosis: assume $\Sigma_{o} \subseteq \Sigma$ is the set of observable events in $A$, and $A$ is $\left(\Delta, \Sigma_{o}\right)$ diagnosable. To build a $\Delta$-diagnoser we proceed as follows [21]13]:

1. build $A^{f}$ as before and replace the events in $\Sigma \backslash \Sigma_{o}$ by $\tau$; recall that $f$ is also replaced by $\tau$ in $A^{f}$ and a boolean value indicates whether a fault has occurred;

2. determinize $A^{f}$ and obtain $B$;

3. define the set of final states $F_{B}$ of $B$ by: $S=\left\{s_{1}, s_{2}, \cdots, s_{l}\right\}$ is in $F_{B}$ iff for each $1 \leq i \leq l, s_{i}$ is a faulty state of $A^{f}$;

4. a $\left(\Delta, \Sigma_{o}\right)$-diagnoser $D$ for $A$ can be constructed as follows:

(a) let $\varrho$ be a run of $A$ and $w=\pi_{\Sigma_{o}}(\operatorname{tr}(\varrho))$.

(b) if when reading $w, B$ reaches a state in $F_{B}$, define $D(w)=1$,

(c) otherwise $D(w)=0$.

Applying this construction for each $\Sigma_{o}=\Sigma_{i}, 1 \leq i \leq n$, we obtain a tuple $\bar{D}=$ $\left(D_{1}, D_{2}, \cdots, D_{n}\right)$ of diagnosers $D_{i}$ which is a $(\Delta, \mathcal{E})$-codiagnoser for $A$. Note that the size of $\bar{D}$ is exponential in the size of $A$ (this is already the case for the diagnosis problem).

\subsection{Synthesis for Timed Automata}

The synthesis of a diagnoser for timed automata [19] is already more complicated than for FA. Timed automata are not (always) determinizable 2 and thus we cannot use the same procedure as for FA and determinize $A^{f}$. Moreover, checking whether a TA is determinizable is not decidable [11, and it is thus impossible to check whether we can use the same procedure.

The construction of a diagnoser for timed automata [19] consists in computing on-the-fly the current possible states of the timed automaton $A^{f}$ after reading a 
timed word $w$. This procedure is effective but gives a diagnoser which is a Turing machine. The machine computes a state estimate of $A$ after each observable event, and if it contains only faulty states, it announces a fault.

Obviously the same construction can be carried out for codiagnosis: we build $M_{i}, 1 \leq i \leq n$ Turing machines that estimate the state of $A$. When one $M_{i}$ 's estimate on an input $\Sigma_{i}$-trace $w$ contains only faulty states, we set $D_{i}(w)=1$ and 0 otherwise. This tuple of Turing machines is a $(\Delta, \mathcal{E})$-codiagnoser.

Computing the estimates with Turing machines might be too expensive to be implemented at runtime. More efficient and compact codiagnosers might be needed with reasonable computation times. In the next section, we address the problem of codiagnosis for TA under bounded resources.

\section{Codiagnosis with Deterministic Timed Automata}

The fault diagnosis problem using timed automata has been introduced and solved by P. Bouyer et al. in [5]. The problem is to determine, given a TA $A$, whether there exists a diagnoser $D$ for $A$, that can be represented by a deterministic timed automaton.

We recall the result of 5 and after we study the corresponding problem for codiagnosis.

\subsection{Fault Diagnosis with Deterministic Timed Automata}

When synthesizing (deterministic) timed automata, an important issue is the amount of resources the timed automaton can use: this can be formally defined 6 by the (number of) clocks, $Z$, that the automaton can use, the maximal constant max, and a granularity $\frac{1}{m}$. As an example, a TA of resource $\mu=\left(\{c, d\}, 2, \frac{1}{3}\right)$ can use two clocks, $c$ and $d$, and the clocks constraints using the rationals $-2 \leq k / m \leq 2$ where $k \in \mathbb{Z}$ and $m=3$. A resource $\mu$ is thus a triple $\mu=\left(Z\right.$, $\left.\max , \frac{1}{m}\right)$ where $Z$ is finite set of clocks, $\max \in \mathbb{N}$ and $\frac{1}{m} \in \mathbb{Q}_{>0}$ is the granularity. DTA $\mu$ is the class of DTA of resource $\mu$.

Remark 2. Notice that the number of locations of the DTA in DTA $A_{\mu}$ is not bounded and hence this family has an infinite (yet countable) number of elements.

If a TA $A$ is $\Delta$-diagnosable with a diagnoser that can be represented by a DTA $D$ with resource $\mu$, we say that $A$ is $(\Delta, D)$-diagnosable. P. Bouyer et al. in $[5$ ] considered the problem of deciding whether there exists a diagnoser which is a DTA with resource $\mu$ :

Problem 7 ( $\triangle$-DTA-Diagnosability [5])

InPUTS: $A$ TA $A=\left(L, l_{0}, X, \Sigma_{\tau, f}, E\right.$, Inv $), \Delta \in \mathbb{N}$, a resource $\mu=\left(Z, \max , \frac{1}{m}\right)$. Problem: Is there any $D \in D T A_{\mu}$ s.t. $A$ is $(\Delta, D)$-diagnosable?

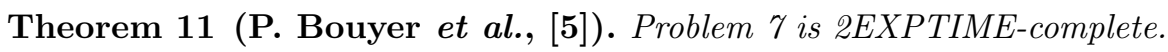


The solution to the previous problem is based on the construction of a twoplayer game, the solution of which gives the set of all $\mathrm{DTA}_{\mu}$ diagnosers (the most permissive diagnosers) which can diagnose $A$ (or $\varnothing$ is there is none).

Let $A=\left(L, l_{0}, X, \Sigma_{\tau, f}, E\right.$, Inv $)$ be a TA, $\Sigma_{o} \subseteq \Sigma$. Define $A(\Delta)=\left(L_{1} \cup L_{2} \cup\right.$ $L_{3}, l_{0}^{1}, X \cup\{z\}, \Sigma_{\tau, f}, \rightarrow_{\Delta}$, Inv $\left._{\Delta}\right)$ as follows:

- $L_{i}=\left\{\ell^{i}, \ell \in L\right\}$, for $i \in\{1,2,3\}$, i.e., $L_{i}$ elements are copies of the locations in $L$,

$-z$ is a (new) clock not in $X$,

- for $\ell \in L, \operatorname{Inv}\left(\ell^{1}\right)=\operatorname{Inv}(\ell), \operatorname{Inv}\left(\ell^{2}\right)=\operatorname{Inv}(\ell) \wedge z \leq \Delta$, and $\operatorname{Inv}\left(\ell^{3}\right)=$ TRUE,

- the transition relation is given by:

- for $i \in\{1,2,3\}, \ell^{i} \stackrel{(g, a, R)}{\longrightarrow} \Delta \ell^{\prime i}$ if $a \neq f$ and $\ell \stackrel{(g, a, R)}{\longrightarrow} \ell^{\prime}$,

- for $i \in\{2,3\}, \ell^{i} \stackrel{(g, f, R)}{\longrightarrow} \Delta \ell^{\prime i}$ if $a \neq f$ and $\ell \stackrel{(g, f, R)}{\longrightarrow} \ell^{\prime}$,

- $\ell^{1} \stackrel{(g, f, R \cup\{z\})}{\longrightarrow} \Delta \ell^{\prime 2}$ if $a \neq f$ and $\ell \stackrel{(g, f, R)}{\longrightarrow} \ell^{\prime}$,

- $\ell^{2} \stackrel{(z=\Delta, \tau, \varnothing)}{\longrightarrow} \ell^{3}$.

The previous construction creates 3 copies of $A$ : the system starts in copy 1 , when a fault occurs it switches to copy 2 , resetting the clock $z$, and when in copy 2 (a fault has occurred) it can switch to copy 3 after $\Delta$ time units (copy 3 could be replaced by a special location $B a d$ ). We can then define $L_{1}$ as the non-faulty locations, and $L_{3}$ as the $\Delta$-faulty locations.

Given a resource $\mu=\left(Y, \max , \frac{1}{m}\right)(X \cap Y=\varnothing)$, a minimal guard for $\mu$ is a guard which defines a region of granularity $\mu$. The (symbolic) universal automaton $\mathcal{U}=\left(\{0\},\{0\}, Y, \Sigma, E_{\mu}\right.$, Inv $\left._{\mu}\right)$ is specified by:

$-\operatorname{Inv}_{\mu}(0)=$ TRUE,

- $(0, g, a, R, 0) \in E_{\mu}$ for each $(g, a, R)$ s.t. $a \in \Sigma, R \subseteq Y$, and $g$ is a minimal guard for $\mu$.

$\mathcal{U}$ is finite because $E_{\mu}$ is finite. Nevertheless $\mathcal{U}$ is not deterministic because it can choose to reset different sets of clocks $Y$ for a pair "(guard, letter)" $(g, a)$. To diagnose $A$, we have to find when a set of clocks has to be reset. This can provide enough information to distinguish $\Delta$-faulty words from non-faulty words.

The algorithm of [5] requires the following steps:

1. define the region graph $R G(A(\Delta) \times \mathcal{U})$,

2. compute a projection of this region graph:

- let $(g, a, R)$ be a label of an edge in $R G(A(\Delta) \times \mathcal{U})$,

- let $g^{\prime}$ be the unique minimal guard s.t. $\llbracket g \rrbracket \subseteq \llbracket g^{\prime} \rrbracket$;

- let $p_{\mathcal{U}}$ be the projection defined by $p_{\mathcal{U}}(g, a, R)=\left(g^{\prime}, a, R \cap Y\right)$ if $a \in \Sigma_{o}$ and $p_{\mathcal{U}}(g, a, R)=\tau$ otherwise.

The projected automaton $p_{\mathcal{U}}(R G(A(\Delta) \times \mathcal{U}))$ is the automaton $R G(A(\Delta) \times \mathcal{U})$ where each label $\alpha$ is replaced by $p_{\mathcal{U}}(\alpha)$.

3. determinize $p_{\mathcal{U}}\left(R G(A(\Delta) \times \mathcal{U})\right.$ ) (removing $\tau$ actions) and obtain $H_{A, \Delta, \mu}$.

4. build a two-player safety game $G_{A, \Delta, \mu}$ as follows:

- each transition $s \stackrel{(g, a, Y)}{\longrightarrow} s^{\prime}$ in $H_{A, \Delta, \mu}$ yields a transition in $G_{A, \Delta, \mu}$ of the form: 


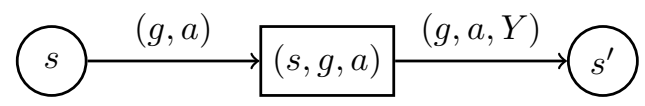

- the round-shaped state are the states of Player 1, whereas the squareshaped states are Player 0 states (the choice of the clocks to reset).

- the bad states (for Player 0 ) are the states $\left\{\left(\ell_{1}, r_{1}\right), \cdots,\left(\ell_{k}, r_{k}\right)\right\}$ with both a $\Delta$-faulty (in $L_{3}$ ) and a non-faulty (in $L_{1}$ ) location. We let $B a d$ denote the set of bad states.

The main results of [5] are:

- there is a TA $D \in \mathrm{DTA}_{\mu}$ s.t. $A$ is $(\Delta, D)$-diagnosable iff Player 0 can win the safety game "avoid Bad" $G_{A, \Delta, \mu}$;

- it follows that Problem 7 can be solved in 2EXPTIME as $G_{A, \Delta, \mu}$ has size doubly exponential in $A, \Delta$ and $\mu$;

- a witness diagnoser $D$ of size doubly exponential in $A, \Delta$ and $\mu$ can be obtained: it is deterministic timed automaton with a set of accepting locations $F$. When the projection $w$ of timed word of $A$ onto $\Sigma_{o}$ is accepted by $D, D$ outputs 1 otherwise it outputs 0 ;

- the acceptance problem for Alternating Turing machines of exponential space can be reduced to Problem 7 and thus it is 2EXPTIME-hard.

Another result of [5] is that for Event Recording Automata (ERA), Problem 7 is PSPACE-complete.

\subsection{Algorithm for Codiagnosability}

In this section we include the alphabet $\Sigma$ a DTA can monitor in the resource $\mu$ and write $\mu=\left(\Sigma, Z, \max , \frac{1}{m}\right)$.

\section{Problem 8 ( $\Delta$-DTA-Codiagnosability)}

InPUTS: $A$ TA $A=\left(L, l_{0}, X, \Sigma_{\tau, f}, E\right.$, Inv $), \Delta \in \mathbb{N}$, and a family of resources $\mu_{i}=\left(\Sigma_{i}, Z_{i}, \max _{i}, \frac{1}{m_{i}}\right), 1 \leq i \leq n$ with $\Sigma_{i} \subseteq \Sigma$.

Problem: Is there any codiagnoser $\bar{D}=\left(D_{1}, D_{2}, \cdots, D_{n}\right)$ with $D_{i} \in D T A_{\mu_{i}}$ s.t. $A$ is $(\Delta, \bar{D})$-codiagnosable?

To solve Problem [8, we extend the previous algorithm for DTA-diagnosability. Let $G^{i}$ be the game $G_{A, \Delta, \mu_{i}}$ and $B a d_{i}$ the set of bad states. Given a strategy $f_{i}$, we let $f_{i}\left(G^{i}\right)$ be the outcom 5 of $G^{i}$ when $f_{i}$ is played by Player 0 . Given $w \in T W^{*}(\Sigma)$ and a DTA $A$ on $\Sigma$, we let $\operatorname{last}(w, A)$ be the location reached when $w$ is read by $A$.

Lemma 6. $A$ is $(\Delta, \bar{D})$-codiagnosable iff there is a tuple of strategies $\bar{f}$ s.t.

(1) $\forall 1 \leq i \leq n, \bar{f}[i]$ is state-based on the game $G^{i}$, and

(2) $\forall w \in \operatorname{Tr}(A) \quad\left\{\begin{array}{l}\text { If } S_{i}=\operatorname{last}\left(\boldsymbol{\pi}_{\Sigma_{i}}(w), f_{i}\left(G^{i}\right)\right), 1 \leq i \leq n, \\ \text { then } \exists 1 \leq j \leq n, \text { s.t. } S_{j} \notin \text { Bad }_{j} .\end{array}\right.$

\footnotetext{
${ }^{5} f_{i}\left(G^{i}\right)$ is a timed transition system.
} 
Item (2) of Lemma 6 states that there is no word in $A$ for which all the Player 0 in the games $G^{i}$ are in bad states. The strategies for each Player 0 are not necessarily winning in each $G^{i}$, but there is always one Player 0 who has not lost the game $G^{i}$.

Proof.

If part. Assume there is a tuple of state-based strategies $\bar{f}=\left(f_{1}, f_{2}, \cdots, f_{n}\right)$ on each game $G^{i}$, s.t. (2) is satisfied. From (1), each choice of Player 0 in $G^{i}$ determines one transition from each square state (see the definition of $G^{i}$ and square states in section 6.1). Thus the graph of $G^{i}$ can be folded into a set of transitions $q \stackrel{g, a, Y}{\longrightarrow} q^{\prime}$ if the choice of Player 0 is $g, a, Y$ in square state $(q, g, a)$. This gives a DTA $G^{i, c}$. We can then build a diagnoser $D_{i}$ defined by the DTA as follows: $(i)$ for each state $q=\left\{\left(\ell_{1}, r_{1}\right), \cdots,\left(\ell_{k}, r_{k}\right)\right\}$ in $G^{i, c}$, if all the $\ell_{j}$ are $\Delta$-faulty, $q$ is accepting; (ii) given $w \in \operatorname{Tr}(A)$, if $\boldsymbol{\pi}_{\Sigma_{i}}(w) \in \mathcal{L}\left(G^{i, c}\right)$, let $D_{i}\left(\boldsymbol{\pi}_{\Sigma_{i}}(w)\right)=1$ and otherwise $0 . \bar{D}$ is a $\Delta$-codiagnoser for $A$. Indeed, let $w \in \operatorname{NonFaulty}^{\operatorname{tr}}(A)$. In each game $G^{i, c}$, we cannot reach a $\Delta$-faulty state because of (2). Hence $\sum_{i=1}^{n} \bar{D}[i]=0$. Now assume $w \in$ Faulty $_{>\Delta}^{t r}(A)$ : In each $G^{i, c}$ we must reach a state $q_{i}$ containing a $\Delta$-faulty state. By (2), there is some $j$ s.t. $q_{j} \notin B a d_{j}$ and this implies that $q_{j}$ is made only of $\Delta$-faulty states and $q_{j}$ is accepting, thus $\bar{D}[j]\left(\boldsymbol{\pi}_{\Sigma_{j}}(w)\right)=1$.

Only If part. For this part we first show that a tuple of strategies $\bar{f}$ exists and then address the state-based problem. Let $\bar{D}=\left(D_{1}, D_{2}, \cdots, D_{n}\right)$ be the tuple of DTA that diagnoses $A$. For each game $G^{i}$, define the strategy $f_{i}$ by: let $\varrho=$ $\left(g_{1}, \lambda_{1}\right)\left(g_{1}, \lambda_{1}, Y_{1}\right)\left(g_{2}, \lambda_{2}\right)\left(g_{2}, \lambda_{2}, Y_{2}\right) \cdots\left(g_{k}, \lambda_{k}\right)$ be a run in $G^{i} ; f_{i}(\varrho)=(g, a, Y)$ if in $D_{i}$ the symbolic sequence $\left(g_{1}, \lambda_{1}\right) \cdots\left(g_{k}, \lambda_{k}\right)$ reaches a location $\ell$ and there is a transition $\left(\ell,(g, a, Y), \ell^{\prime}\right)$ in $D_{i}$. By assumption, as $\bar{D}$ is a $\Delta$-codiagnoser, for each $w \in$ Faulty $_{>\Delta}^{\operatorname{tr}}(A)$, there is at least one $D_{j}$ which reaches an accepting state after reading $\boldsymbol{\pi}_{\Sigma_{j}}(w)$.

As a consequence, in the corresponding game, $G^{j}$, the state reached is made only of $\Delta$-faulty states. Indeed, if a non-faulty state is reachable, then the word $w$ is also the projection of a non faulty run. Hence $D_{j}$ should announce 0 which is a contradiction.

If $w \in$ NonFaulty $^{t r}(A)$, all the states reached in each $G^{i}$ are non faulty.

Now assume we have the strategies $f_{i}, 1 \leq i \leq n$. We can construct statebased strategies on each game $G^{i}$. Given $f_{1}$, (not necessarily winning) on $G^{1}$, let $T_{1}$ be the set of bad states reachable in $f_{1}\left(G^{1}\right)$. Define the language $\mathcal{L}_{1}$ to be the set of words $w \in \operatorname{Tr}(A)$ s.t. a state in $T_{1}$ is reachable in $f_{1}\left(G^{1}\right)$ when reading $\boldsymbol{\pi}_{\Sigma_{1}}(w)$. These are the words on which $f_{1}$ is not winning in $G^{1}$.

Let Reach $\left(f_{1}\left(G^{1}\right)\right)$ be the set of states reachable in $G^{1}$. There is a strategy $\left(f_{1}\right)$ to avoid $B_{1}=\operatorname{Reach}\left(G^{1}\right) \backslash \operatorname{Reach}\left(f_{1}\left(G^{1}\right)\right)$. Hence there is a state-based strategy $f_{1}^{\prime}$ that avoids $B_{1}$.

Let $1 \leq i<n$. Consider the game $f_{i+1}\left(G^{i+1}\right)$ restricted to the (projections of the) words $w \in \mathcal{L}_{i}$. The idea is that on $\mathcal{L}_{i}$, a strategy $f_{j}, j \leq i$ is winning in $G^{j}$. In this restricted game, we define the set $T_{i+1}$ of bad states that are still 
reachable. Let $\mathcal{L}_{i+1}$ be the set of words $w \in \operatorname{Tr}(A)$ s.t. a state in $T_{i+1}$ is reachable in the restricted timed transition system $f_{i+1}\left(G^{i+1}\right)$.

Notice that we can construct a state-based strategy $f_{i}^{\prime}$ which avoids the same states as $f_{i}$ does. For each restricted game $f_{i}^{\prime}\left(G^{i}\right)$ we define the diagnoser $D_{i}$ as before. If for some $i, \mathcal{L}_{i}=\varnothing$, we can define the diagnosers $D_{k}, k \geq i$ to always announce 0 for each word.

The tuple $\overline{f^{\prime}}$ is a $(\Delta, \mathcal{E})$-codiagnoser for $A$ and all the $\overline{f^{\prime}}[i]$ are state-based on $G^{i}$.

From the previous Lemma, we can obtain the following result:

Theorem 12. Problem 8 is 2EXPTIME-complete.

Proof. 2EXPTIME-hardness follows from Theorem 11, from [5]. 2EXPTIME easiness is obtained using the following algorithm:

1. compute the games $G^{i}, 1 \leq i \leq n$;

2. select a state-based strategy on each game $G^{i}$;

3. check condition (2) of Lemma 6 .

The sizes of the games $G^{i}$ are doubly exponential in $A, \Delta$ and the resources $\mu_{i}$ (recall that $\Sigma_{i}$ is included in $\mu_{i}$ ). There is a doubly exponential number of state-based strategies for each game $G^{i}$. Once selected we have a DTA $G^{i, c}$.

Checking condition (2) of Lemma 6 can be done on the product $A(\Delta) \times$ $G^{1, c} \times \cdots \times G^{n, c}$. It amounts to deciding whether a location in $L_{3} \times \operatorname{Bad}_{1} \times$ $\cdots \mathrm{Bad}_{n}$ is reachable. Reachability can be checked in PSPACE for product of TA (Theorem 2). As the size of the input is doubly exponentian in the size of $A$, this results in a 2 EXPSPACE algorithm.

Nevertheless, there is no exponential blow up in the number of clocks of the product. Actually the size of $R G\left(A(\Delta) \times G^{1, c} \times \cdots \times G^{n, c}\right)$ is $|L| \cdot 2^{2^{|A|+\left|\mu_{1}\right|}} \cdots \cdots$ $2^{2^{|A|+\left|\mu_{n}\right|}} \cdot(n \cdot|X|) ! \cdot 2^{n \cdot|X|} \cdot K^{n \cdot|X|}$ with $K$ the maximal constant in $A, \Delta$, and the resources $\mu_{i}$. It is doubly exponential in the size of $A, \Delta$ and the resources $\mu_{i}$. Reachability can be checked in linear time on this graph and thus in doubly exponential time in the size of $A, \Delta$ and the resources. Step 3 above is done at most a doubly exponential number of times

and the result follows.

\section{Conclusion \& Future Work}

Table 1 gives an overview of the results described in this paper (bold face) for the codiagnosis problems in comparison with the results for the diagnosis problems (second line, normal face).

Our ongoing work is to extend the results on diagnosis using dynamic observers [98] to the codiagnosis framework. 


\begin{tabular}{||c|c|c|c|c||}
\hline \hline & $\Delta$-Codiagnos. & Codiagnosability & Optimal Delay & $\begin{array}{c}\text { Synthesis } \\
\text { (Bounded Resources) }\end{array}$ \\
\hline \hline \multirow{2}{*}{ FA } & PSPACE-C. & PSPACE-C. & PSPACE & EXPTIME \\
& PTIME [21 13] & PTIME [21[13] & PTIME [21[13] & EXPTIME [18] \\
\hline \hline \multirow{2}{*}{ TA } & PSPACE-C. & PSPACE-C. & PSPACE & 2EXPTIME-C. \\
& PSPACE-C. [19] & PSPACE-C. [19] & PSPACE [7] & 2EXPTIME-C. [5] \\
\hline \hline
\end{tabular}

Table 1. Summary of the Results

\section{References}

1. Luca Aceto and François Laroussinie. Is your model checker on time? on the complexity of model checking for timed modal logics. J. Log. Algebr. Program., 52-53:7-51, 2002.

2. Rajeev Alur and David Dill. A theory of timed automata. Theoretical Computer Science, 126:183-235, 1994.

3. João Carlos Basilio and Stéphane Lafortune. Robust codiagnosability of discrete event systems. In IEEE Computer Society, editor, Proceedings of the American Control Conference (ACC'09), pages 2202-2209, 2009.

4. Gerd Behrmann, Alexandre David, and Kim G. Larsen. A tutorial on UppaAL. In Marco Bernardo and Flavio Corradini, editors, Formal Methods for the Design of Real-Time Systems: 4th International School on Formal Methods for the Design of Computer, Communication, and Software Systems, SFM-RT 2004, volume 3185 of $L N C S$, pages 200-236. Springer Verlag, September 2004.

5. Patricia Bouyer, Fabrice Chevalier, and Deepak D'Souza. Fault diagnosis using timed automata. In Vladimiro Sassone, editor, Proceedings of the 8th International Conference on Foundations of Software Science and Computation Structures (FoSSaCS'05), volume 3441 of LNCS, pages 219-233, Edinburgh, U.K., April 2005. Springer Verlag.

6. Patricia Bouyer, Deepak D'Souza, P. Madhusudan, and Antoine Petit. Timed control with partial observability. In Warren A. Hunt, Jr and Fabio Somenzi, editors, Proceedings of the 15th International Conference on Computer Aided Verification (CAV'03), volume 2725 of $L N C S$, pages 180-192, Boulder, Colorado, USA, July 2003. Springer.

7. Franck Cassez. A Note on Fault Diagnosis Algorithms. In 48th IEEE Conference on Decision and Control and 28th Chinese Control Conference, Shanghai, P.R. China, December 2009. IEEE Computer Society.

8. Franck Cassez and Stavros Tripakis. Fault diagnosis with static or dynamic diagnosers. Fundamenta Informaticae, 88(4):497-540, November 2008.

9. Franck Cassez, Stavros Tripakis, and Karine Altisen. Sensor minimization problems with static or dynamic observers for fault diagnosis. In 7th Int. Conf. on Application of Concurrency to System Design (ACSD'07), pages 90-99. IEEE Computer Society, 2007.

10. Rami Debouk, Stéphane Lafortune, and Demosthenis Teneketzis. Coordinated decentralized protocols for failure diagnosis of discrete event systems. Discrete Event Dynamic Systems, 10(1-2):33-86, 2000.

11. Olivier Finkel. On decision problems for timed automata. Bulletin of the European Association for Theoretical Computer Science, 87:185-190, 2005. 
12. Gerard J. Holzmann. Software model checking with spin. Advances in Computers, 65:78-109, 2005.

13. Shengbing Jiang, Zhongdong Huang, Vigyan Chandra, and Ratnesh Kumar. A polynomial algorithm for testing diagnosability of discrete event systems. IEEE Transactions on Automatic Control, 46(8), August 2001.

14. Dexter Kozen. Lower bounds for natural proof systems. In FOCS, pages 254-266. IEEE, 1977.

15. Wenbin Qiu and Ratnesh Kumar. Decentralized failure diagnosis of discrete event systems. IEEE Transactions on Systems, Man and Cybernetics, Part A: Systems and Humans, 36(2):384-395, 2006.

16. P.J.G. Ramadge and W.M. Wonham. Supervisory control of a class of discrete event processes. SIAM Journal of Control and Optimization, 25(1):1202-1218, 1987.

17. P.J.G. Ramadge and W.M. Wonham. The control of discrete event systems. Proc. of the IEEE, 77(1):81-98, 1989 .

18. Meera Sampath, Raja Sengupta, Stephane Lafortune, Kasim Sinnamohideen, and Demosthenis C. Teneketzis. Diagnosability of discrete event systems. IEEE Transactions on Automatic Control, 40(9), September 1995.

19. Stavros Tripakis. Fault diagnosis for timed automata. In Werner Damm and ErnstRüdiger Olderog, editors, Proceedings of the International Conference on Formal Techniques in Real Time and Fault Tolerant Systems (FTRTFT'02), volume 2469 of $L N C S$, pages 205-224. Springer Verlag, 2002.

20. Yin Wang, Tae-Sic Yoo, and Stéphane Lafortune. Diagnosis of discrete event systems using decentralized architectures. Discrete Event Dynamic Systems, 17(2):233-263, 2007.

21. Tae-Sic Yoo and Stéphane Lafortune. Polynomial-time verification of diagnosability of partially-observed discrete-event systems. IEEE Transactions on Automatic Control, 47(9):1491-1495, September 2002. 